\title{
MRS Photodiode in Strong Magnetic Field
}

\author{
D. Beznosko, G. Blazey, A. Dyshkant, K. Francis, D. Kubik, V. Rykalin, M. A. Tartaglia, V. Zutshi
}

\begin{abstract}
The experimental results on the performance of the MRS (Metal/Resistor/Semiconductor) photodiode in the strong magnetic field of 4.4T, and the possible impact of the quench of the magnet at 4.5T on sensor's operation are reported.
\end{abstract}

\section{INTRODUCTION}

Future detectors (like Digital Hadron Calorimeter [1] [2] for a future $\mathrm{e}^{+} \mathrm{e}^{-}$linear collider) may require the use of scintillating cells with embedded photodetectors, immersed in a strong magnetic field. This imposes constraints on the performance of the photodetectors, which has directed our attention to the latest developments in the field of solid-state photo multipliers working in avalanche mode [3].

In this paper we have concentrated on the possible issues of the MRS output in the presence of strong magnetic field; i.e., the dependence of output's amplitude, area and rise time on the field, and the effects of magnet's quench on the sensor.

\section{DETECTOR DESCRIPTION AND OPERATIONAL PRINCIPLES}

The MRS photodiode is a multi-pixel solid-state device with every pixel operating in the limited Geiger multiplication mode. A resistive layer on the sensor surface achieves avalanche quenching. The devices tested were of round shape, and they had 1000 pixels per $1.1 \mathrm{~mm}$ diameter photosensitive area, with the quantum efficiency (QE) of the device reaching $25 \%$ at $500 \mathrm{~nm}[4]$. Due to the fact that the thickness of the active layer of this sensor is about 7 microns, MRS is theoretically expected to be non-sensitive to the magnetic field.

\section{EXPERIMENTAL SECTION}

\section{A. Magnet Description}

The magnet used was a standard Tevatron Dipole [5], whose field strength was $\sim 1$ Tesla per kA. The sensors were placed in the body of the magnet (far from the ends), where the field is

Manuscript received December 14, 2004. This work was supported in part by the U.S. Department of Education under Grant No. P116Z010035, the Department of Energy, and the State of Illinois Higher Education Cooperation Act.

M.A. Tartaglia is with Fermi National Accelerator Laboratories, Batavia, IL 60510 USA (telephone: 630-840-3890, email: tartaglia@fnal.gov).

D. Beznosko, G. Blazey, A. Dyshkant, K. Francis, D. Kubik, V. Rykalin, V. Zutshi are with Northern Illinois University, DeKalb, IL 60115 USA (telephone: 815-753-3504, email: dyshkant@fnal.gov). very uniform (at the level of 1 part in 10000). The dipole field direction is vertical. The temperature in the magnet aperture is not cryogenic and is close to "room" temperature. The following characterizes the speed with which magnetic field collapses during a quench event: the current in the magnet decays (approximately) exponentially with a time constant of about 0.25 seconds (determined by the $L / R$ of the circuit that are not adjustable).

\section{B. MRS Assembly Description}

For this test, five MRS sensors were used, arranged in different directions with respect to the magnetic field. All sensors were biased at $30.0 \mathrm{~V}$ that was well within operating range of all five. An optical splitter was used to deliver similar light pulse to each of the sensors. The light pulse was produced by the UV LED (peak emission $\sim 400 \mathrm{~nm}$ ) [6]. The pulse from the pulse generator was $\sim 30 \mathrm{~ns}$ wide with $\sim 5.5 \mathrm{~V}$ amplitude. The LED was embedded into the $10 \mathrm{~mm}$ thick extruded scintillator [7]. The LED-scintillator part was placed well outside the magnet to avoid any effects of the field. The light pulse from the scintillator to the splitter was carried via $\sim 2.5 \mathrm{~m}$ long, $2 \mathrm{~mm}$ outer diameter, KURARAY [8], multiclad, Y-11, WLS fiber. The output of the sensors was fed into the Agilent [9] Infiniium54832D MSO oscilloscope without additional preamplifier. The schematic of this assembly is given in Fig. 1.

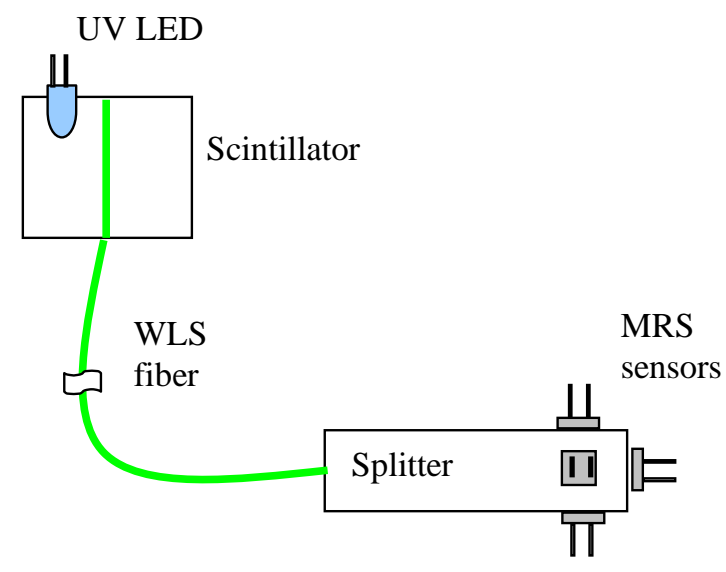

Fig. 1. MRS assembly schematics.

The temperature inside the magnet was measured before and after the tests and was $5.8^{\circ} \mathrm{C} \pm 0.5$. 


\section{Experimental Results}

The data for all 5 channels were obtained. Because of the similarity of the results, data only for channel 4 will be presented for illustrative purposes. This channel corresponds to the MRS sensor that was positioned at the tip of the splitter. The electrons in this sensor move along the same axis inside the dipole as the beam would, therefore the MRS in channel 4 should experience the biggest effects of the B field, if any.

The following characteristics of the sensor's output were measured: amplitude, area, and rise time. Measurements were carried out at 0T, 4.0066T, 0T, 4.0065T, 0T and 4.4077T. The repeated measurements at $0 \mathrm{~T}$ are conducted in order to eliminate unknown factors like the possible temperature changes during the experiment. In addition, measurements were conducted immediately after magnet quench at 4.5T (the field was zero during these measurements). The pole with the sensors and the LED-scintillator assembly was inserted into the magnet approximately 12 hours before the experiment so that it would be at the same temperature as inside the dipole. A constant stream of nitrogen is pumped through the magnet to remove the humidity.

Fig. 2 is the superposition of the MRS output of channel 4 at 0T, 4T, 4.4T, and after quench at $4.5 \mathrm{~T}$. The scale here is $20 \mathrm{mV}$ per cell on vertical axis and 200ns per cell on horizontal.

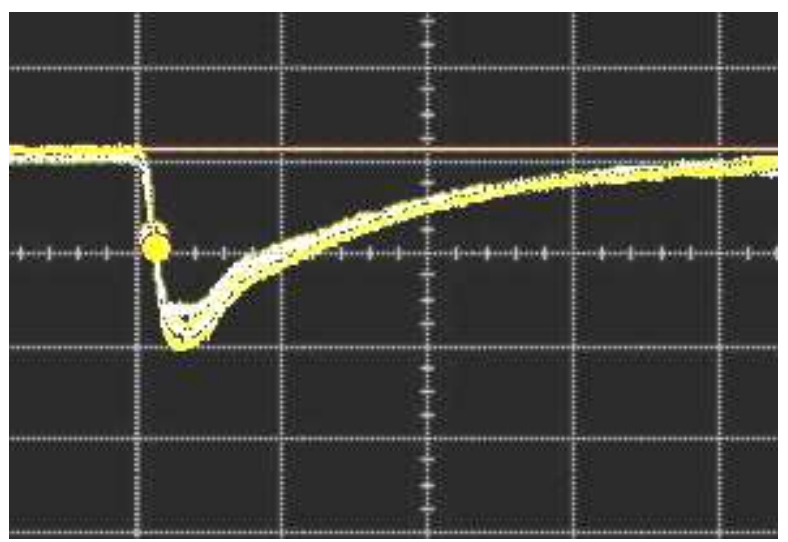

Fig. 2. Superposition of the MRS outputs at the field strengths of 0T, 4T, 4.4T, and after quench at $4.5 \mathrm{~T}$.

From Fig. 2 there are no immediate indications of differences in the output that could be easily seen by the eye. Fig. 3 shows the values of the area of MRS output as a function of the magnetic field strength. The area is a measure of total charge of the output with a $50 \Omega$ load. Each point in every figure is an average of at least few hundred measurements at each field strength value. The errors are given directly by the oscilloscope. Here and in all further plots 4.5qT label indicates a measurement done after the magnet quench at $4.5 \mathrm{~T}$ field. The q-1 and q-1-m labeled data points were taken at 0T some time (approximately 5 and 10 minutes) after the experiment, with the oscilloscope, power supply and signal generator being turned off and back on between q-1 and q-1-m measurements. The biggest difference between points at $0 \mathrm{~T}$ and $4 \mathrm{~T}$ is $\sim 1.5 \%$ that is within the measurement error. However, all the points at $4 \mathrm{~T}$ seem to differ from the 0T ones, whereas all the point measured at 0T have much smaller spread. Other channels do not show this systematic difference of output values.

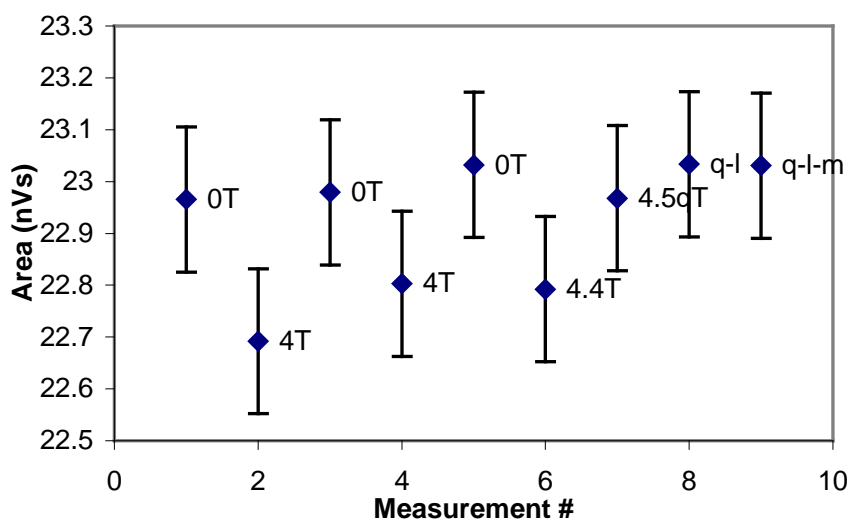

Fig. 3. Area of the MRS output.

Fig. 4 shows the values of the amplitude of MRS output as a function of the magnetic field strength. The amplitude is a measure of the peak current of the output with a $50 \Omega$ load. The maximum of $\sim 1 \%$ change in output amplitude between field strength values $0 \mathrm{~T}$ and $4.4 \mathrm{~T}$ is observed.

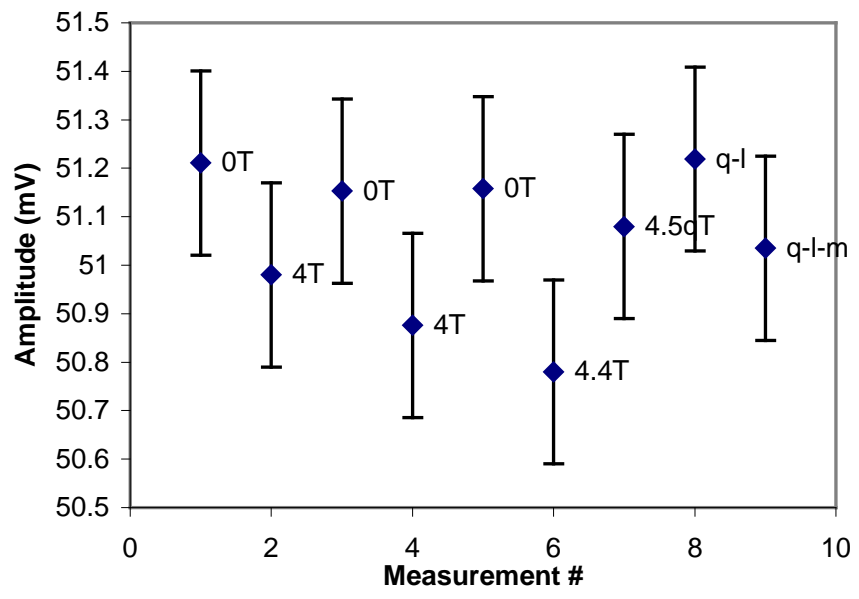

Fig. 4. Amplitude of the MRS output.

The dependence of rise time on the magnetic field strength was also studied (Fig. 5). The behavior of the signal rise time seems to be quite independent on the field strength; however, 
this could be due to the fact that the spread of the values is smaller than the jitter introduced by the way oscilloscope measures the rise time.

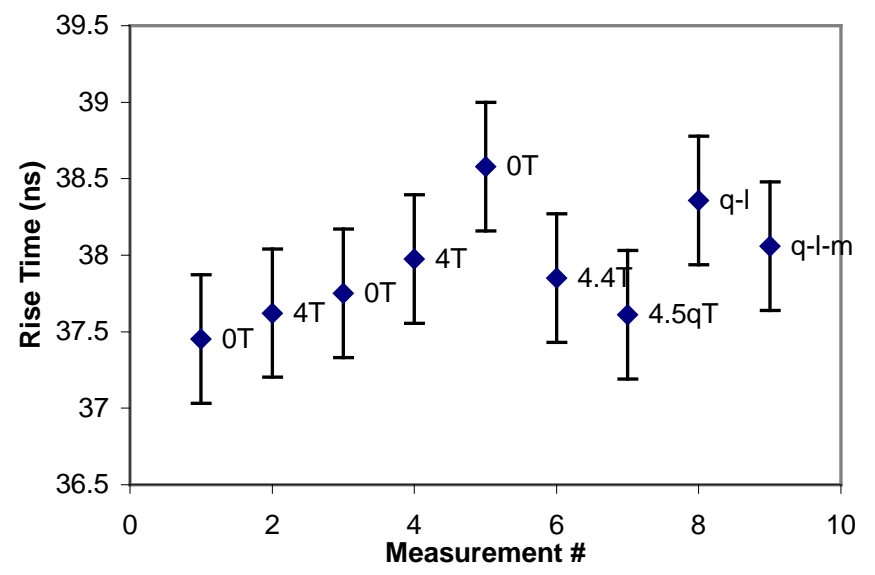

Fig. 5. Rise time of the MRS output.

\section{CONCLUSIONS}

Measurements performed using 5 MRS sensors in the strong magnetic field point to the insensitivity of the sensor's output on the field strength of up to $4.4 \mathrm{~T}$ within $1 \%-1.5 \%$. There are also indications that magnet quench has none or immeasurably small effect on the sensors. However, a small but quite systematic difference of the output values at 0T and 4T for channel 4 (other channels do not show this pattern) may warrant further investigation of this topic, possibly with higher fields strength values.

\section{ACKNOWLEDGEMENT}

The authors would like to thank Boris Baldine for useful advice, and Pat Richards, Larry Gregersen and Phillip Stone for providing excellent mechanical and machining support.

\section{REFERENCES}

[1] A. Dyshkant, D. Beznosko, G. Blazey, D. Chakraborty, K. Francis, D. Kubik et al., "Towards a Scintillator-Based Digital Hadron Calorimeter for the Linear Collider Detector", IEEE vol. 51, no. 4, pp.1590-1595, Aug. 2004.

[2] A. Dyshkant, D. Beznosko, G. Blazey, D. Chakraborty, K. Frances, D. Kubik et al, "Small Scintillating Cells as the Active Elements in a Digital Hadron Calorimeter for the e+e- Linear Collider Detector", FERMILABPUB-04/015, Feb 9, 2004

[3] D. Beznosko, G. Blazey, A. Dyshkant, K. Francis, D. Kubik, A. PlaDalmau et al., "MRS Photodiode", FERMILAB-CONF-04-210-E, September 15, 04
[4] M. Golovin, A.V. Akindinov, E.A. Grigorev, A.N. Martemyanov, P.A. Polozov, "New Results on MRS APDS", Nucl. Instrum. Meth. A387 231234, 1997

[5] R. Hanft, B. C. Brown, W. E. Cooper, D. A. Gross, L. Michelotti, E. E. Schmidt et al., "Magnetic Field Properties of Fermilab Energy Saver Dipoles", FERMILAB-TM-1182, March 1983

[6] Bivar Inc., 4 Thomas, Irvine, CA 92618, USA

[7] D. Beznosko, A. Bross, A. Dyshkant, A. Pla-Dalmau V. Rykalin, "FNALNICADD Extruded Scintillator", FERMILAB-CONF-04-216-E, September 15, 04

[8] Kuraray America Inc., 200 Park Ave, NY 10166,USA; 3-1-6, NIHONBASHI, CHUO-KU, TOKYO 103-8254, JAPAN.

[9] Agilent Technologies, Inc. Headquarters, 395 Page Mill Rd., Palo Alto, CA 94306, United States 